\title{
Diagnostic Accuracy of Computed Tomography Scan to Diagnose Subarachnoid Hemorrhage in Patients Presenting with Thunderclap Headache
}

\author{
Sajan Lal ${ }^{1}$, Bhesham Shahani ${ }^{1}$, Maria Hassan ${ }^{1, ~}$, , Ayub Mansoor ${ }^{2}$, Reshma Sajan $^{3}$ \\ ${ }^{1}$ Department of Radiology, Dr Ziauddin Hospital, Clifton, Karachi, Pakistan \\ ${ }^{2}$ Department of Radiology, Liaquat National Hospital, Karachi, Pakistan \\ ${ }^{3}$ Department of Gynaecology and obstetrics, Civil Hospital, Karachi, Pakistan \\ Email address: \\ dr_spunjwani2000@yahoo.com (S.Lal), shahani81@hotmail.com (B. Shahani), misshassan07@hotmail.com (M. Hassan), \\ ayubmansoor@hotmail.com (A. Mansoor), shadanir@yahoo.com (R. Sajan) \\ ${ }^{*}$ Corresponding author
}

\section{To cite this article:}

Sajan Lal, Bhesham Shahani, Maria Hassan, Ayub Mansoor, Reshma Sajan. Diagnostic Accuracy of Computed Tomography Scan to Diagnose Subarachnoid Hemorrhage in Patients Presenting with Thunderclap Headache. International Journal of Chinese Medicine. Vol. 3, No. 1, 2019, pp. 17-21. doi: 10.11648/j.ijcm.20190302.11

Received: May 4, 2019; Accepted: June 11, 2019; Published: June 26, 2019

\begin{abstract}
Introduction: Thunderclap headache (TCH) is a severe headache that peaks within 60 seconds of onset. It's an uncommon type of headache, but recognition and diagnosis are important because of the possibility of a serious underlying brain disorder. Severe headache has long been recognized as a signature feature of subarachnoid hemorrhage (SAH). Lumbar puncture (LP) has the advantage of picking up cases missed on computed tomography (CT) scan, owing to its ability to detect small bleeds directly from the cerebrospinal fluid (CSF). This study can help us in understanding the role of this modality for early diagnosis and therefore timely management of patients. Subject and methods:A retrospective cross-sectional study for a period of six months was conducted at a tertiary care hospital in Karachi, Pakistan. A total of 189 patients presenting with TCH were included in this study. CT was performed on Toshiba Activion 16 slice CT scanner (Toshiba Medical Systems, Otawara, Japan). Presence of SAH was confirmed by CSF analysis after LP. Data were registered on proforma, then transferred to IBM SPSS Statistics for Windows, Version 20.0 (IBM Corp., Armonk, NY) for statistical analysis. Diagnostic accuracy of CT scan in detecting SAH was calculated. Stratification was done on age and sex to see the effect of these modifiers on outcome using chi square test; $\mathrm{p} \leq 0.05$ was considered as significant. Results:The average age of the patients was $47.97 \pm 9.96$ years. Sensitivity, specificity, positive predictive value (PPV), and negative predictive value (NPV) of CT was $88 \%, 91.4 \%, 78.6 \%$, and $95.5 \%$, respectively while diagnostic accuracy of CT scan in the detection of SAH was $90.48 \%$.Conclusion:This study concludes that $\mathrm{CT}$ is the preferred non-invasive approach for the majority of patients who present with suspected SAH.
\end{abstract}

Keywords: Thunderclap Headache, Subarachnoid Haemorrhage, Lumbar Puncture, Computed Tomography

\section{Introduction}

Thunderclap headache $(\mathrm{TCH})$ is a severe headache that peaks within 60 seconds of onset [1]. It's an uncommon type of headache, but recognition and diagnosis are important because of the possibility of a serious underlying brain disorder. Most importantly, subarachnoid hemorrhage (SAH) should be excluded [2].

A foremost consideration in patients presenting with $\mathrm{TCH}$ is the exclusion of spontaneous SAH [3]. Severe headache has long been recognized as the signature feature of SAH. Of patients with severe, abrupt-onset headache $(\mathrm{TCH})$, about $20 \%-50 \%$ have SAH [4].

In one preliminary report of 3,132 neurologically intact patients with the worst headache, only 240 had SAH. The sensitivity of computed tomography (CT) scan overall for 
SAH was $92.6 \%$ and specificity was $95 \%-100 \%$ [5]. In another study, 19 patients out of 25 that had the diagnosis of SAH were sent for CT scan due to clinical suspicion of SAH over a one-year period. The overall sensitivity of CT scan was $76 \%$ [6]. The initial diagnostic test in a patient with suspected SAH is a CT scan of the brain without contrast. In patients suspected of having a $\mathrm{SAH}$, a normal CT should be followed by lumbar puncture (LP) to detect xanthochromia [7].

It is very important to have an early diagnosis of SAH because patients with aneurysmal $\mathrm{SAH}$, even those without additional neurologic deficits on initial presentation, are at risk for re-bleeding and decompensation if the aneurysm is not treated [8]. SAH is missed in $20 \%-50 \%$ of patients at first presentation [9]. SAH is diagnosed by demonstrating blood in the cerebrospinal fluid (CSF) by means of a CT brain scan or LP [9].

LP has the advantage of picking up cases missed on CT scan, owing to its ability to detect small bleeds directly from CSF. It remains the gold standard diagnostic test [10]. The sensitivity of LP to detect SAH approaches $100 \%$ [11].

In most of the developing countries, including Pakistan, literature about the role of CT scan in the early evaluation of patients with $\mathrm{SAH}$ presenting with $\mathrm{TCH}$ is lacking. Therefore, the aim of this study is to determine the diagnostic accuracy of CT scan brain for SAH among patients of the local population; it is a routine practice in hospitals of Pakistan to perform a LP in every patient having suspicion of non-traumatic SAH. LP is a highly invasive procedure, risks of epidural bleed, paraplegia, post procedure headache, etc. are high. If the results of this study are satisfactory, LP should be reserved only for those patients in which there is high suspicion of SAH with negative CT scan findings. This will help us in understanding the role of this modality for early diagnosis and therefore timely management of patients. This will also add to the database which will be further helpful for treatment and management protocol.

\section{Materials \& Methods}

A retrospective cross-sectional study for a period of six months was conducted at a tertiary care hospital in Karachi, Pakistan. A total of 189 patients presenting with TCH were included in this study. Non probability consecutive sampling technique was used. The inclusion criteria included all male and female patients presenting with $\mathrm{TCH}$ with age group ranging from 20-70 years who underwent CT brain without contrast followed by LP. The exclusion criteria included recent head trauma (less than 4 months before evaluation), previous neurosurgical procedure, history of brain tumor, patients with bleeding disorder, patient on anti-platelet drugs and patients who did not give consent for ct scan brain and LP.

Informed consent was waived as it is a retrospective study. Demographics were collected by the principal investigator. Computed tomography was performed on multidetector Asteion 16 (Toshiba Medical Systems, Tokyo, Japan). Scanning protocol includes the acquisition of axial helical CT sections extending from the vertex up to the base of the skull. All the patients had a CT brain without contrast for evaluation of SAH and subsequently undergo CSF analysis. Assessment of SAH on CT scan was done using the console of CT scan by a radiologist with more than five years of experience. Presence of SAH was confirmed by CSF analysis after LP. The LP was performed by a consultant with more than five years of experience. Findings of CT scan and LP were recorded. All the data was collected on a specially designed Proforma by the principal investigator. Confounding variables and biasness was controlled by strictly following the inclusion and exclusion criteria.

Statistical data analysis was done using IBM SPSS Statistics for Windows, Version 20.0 (IBM Corp., Armonk, NY). The qualitative data like demographics (sex; male or female), CT scan findings for SAH (yes/no), LP analysis (positive/negative for blood) were presented as frequency and percentages. Quantitative data in the study like age (in years) was presented as means and standard deviations. The main outcome variable was correct findings on CT scan in evaluation of SAH (yes/no) which was presented as diagnostic accuracy; it was calculated by taking sensitivity, specificity, positive predictive value (PPV), and negative predictive value (NPV), using LP as the gold standard. Stratification was done on age and sex to see the effect of these modifiers on outcome using chi square test; $p \leq 0.05$ was considered as significant.

\section{Results}

A total of 189 patients presenting with thunderclap headache were included in this study. Age distribution is presented in Figure 1.

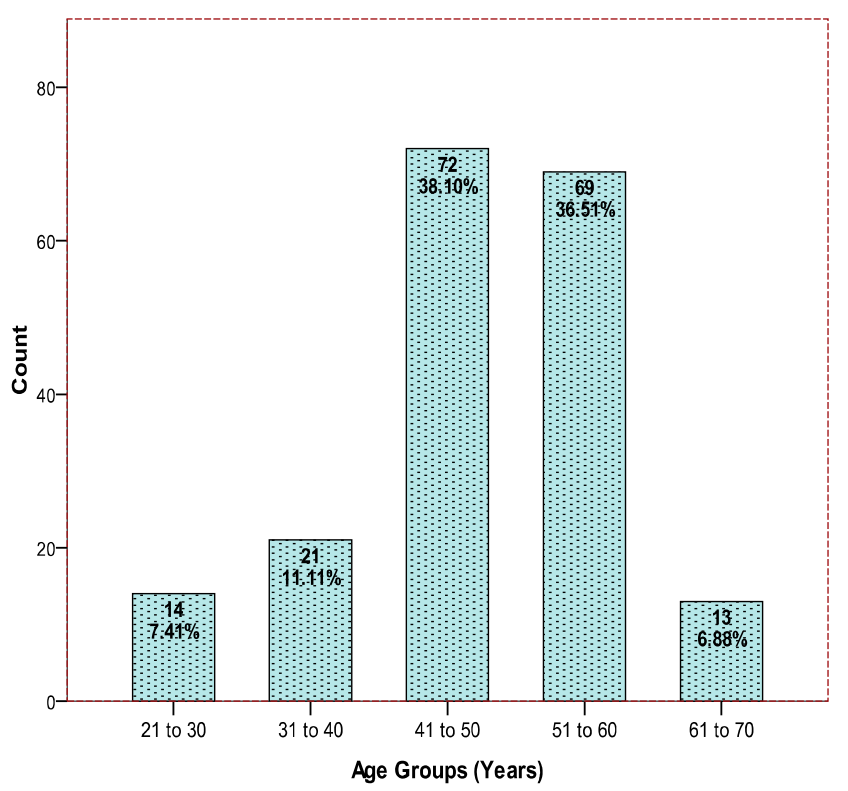

Figure 1. Age distribution of the patients.

The average age of the patients was $47.97 \pm 9.96$ years (95\% confidence interval [CI]: 46.54 to 49.40 ). Out of 189 
cases, $82(43.39 \%)$ were male and 107 (56.51\%) were female. CT scan finding for SAH was positive in $29.6 \%$ $(56 / 189)$ while lumber puncture analysis of the blood finding for SAH was positive in $26.46 \%(50 / 189)$ of the cases (Figures 2-3).

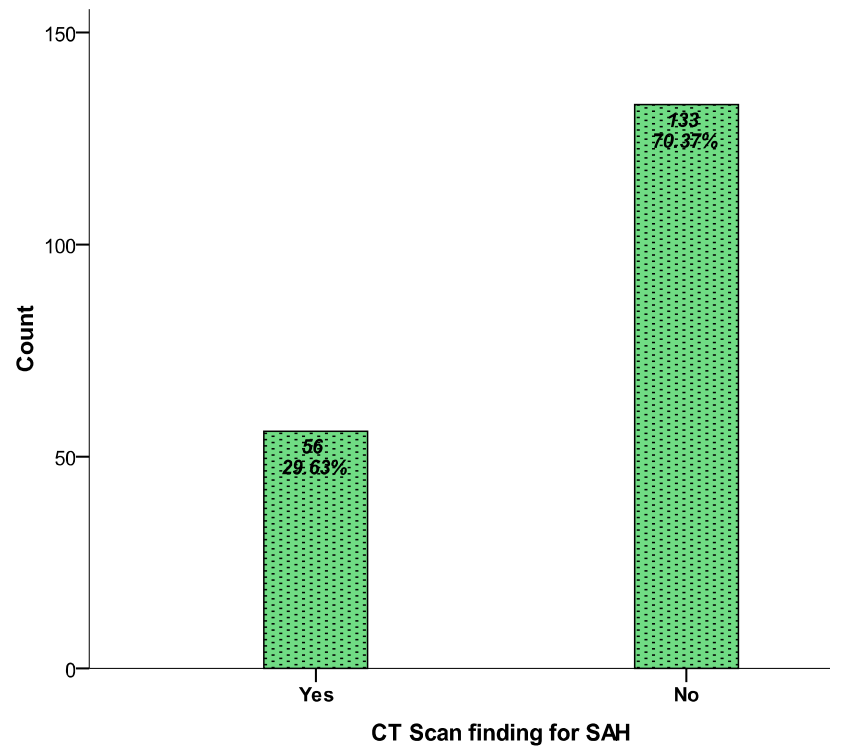

Figure 2. Computed tomography scan finding for subarachnoid haemorrhage (SAH).

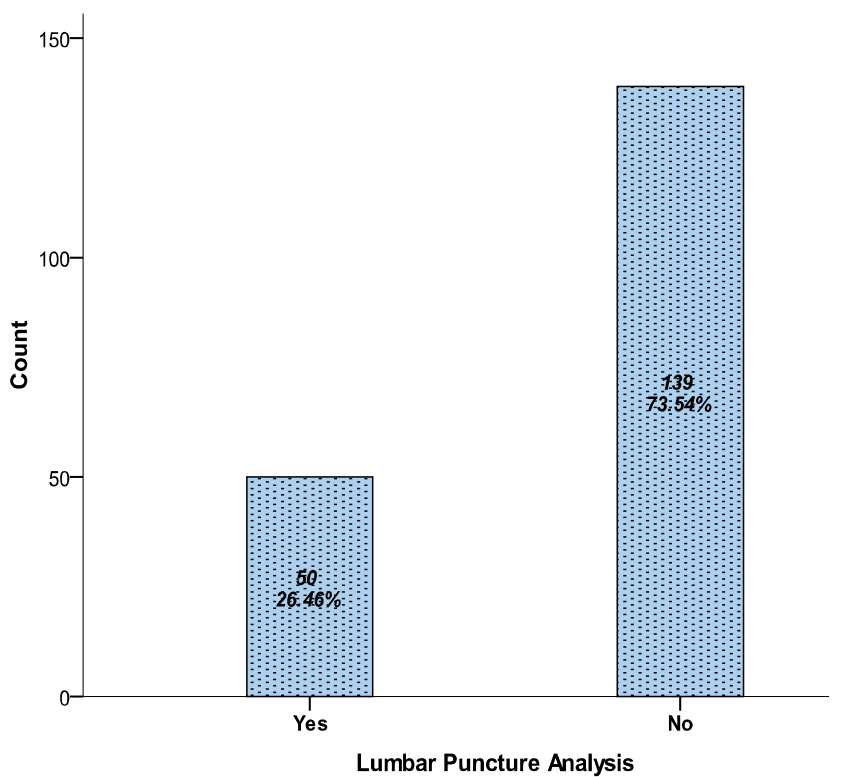

Figure 3. Lumber puncture analysis of the blood for subarachnoid haemorrhage (SAH).

Sensitivity, specificity, PPV, and NPV of CT was $88 \%$, $91.4 \%, 78.6 \%$, and $95.5 \%$ respectively while diagnostic accuracy of CT scan in detection of SAH was $90.48 \%$ $(171 / 189)$ as presented in Table 1.

Table 1. Sensitivity, specificity, PPV, and NPV of CT scan to diagnose SAH in patients presenting with thunderclap headache.

\begin{tabular}{lll}
\hline \multirow{2}{*}{ CT Finding } & Gold Standard LUMBER PUNCTURE & \\
\cline { 2 - 3 } & Positive & Negative \\
\hline Negative & $6(\mathrm{FN})$ & $127(\mathrm{TN})$ \\
Total & $50(26.5 \%)$ & $139(73.5 \%)$ \\
Sensitivity & $=44 / 50$ & $=88 \%$ \\
Specificity & $=127 / 139$ & $=91.4 \%$ \\
PPV & $=44 / 56$ & $=78.6 \%$ \\
NPV & $=127 / 133$ & $=95.5 \%$ \\
\hline
\end{tabular}

PPV: positive predictive value; NPV: negative predictive value; CT: computed tomography; SAH: subarachnoid haemorrhage.

Diagnostic accuracy of CT for below and equal to 50 years of age patients was $88.7 \%$ and for above 50 years of age patients was $92.6 \%$ as presented in Table 2 .

Table 2. Diagnostic accuracy of CT scan to diagnose SAH in patients presenting with thunderclap headache by age groups.

\begin{tabular}{|c|c|c|c|c|c|}
\hline \multirow{2}{*}{ Age Groups } & \multirow{2}{*}{ CT scan } & \multicolumn{2}{|c|}{ Gold Standard LUMBER PUNCTURE } & \multirow{2}{*}{ Total } & \multirow{2}{*}{ P-Value } \\
\hline & & Positive & Negative & & \\
\hline \multirow{3}{*}{$\leq 50$ years } & Positive & $27(\mathrm{TP})$ & $9(\mathrm{FP})$ & 36 & \multirow{3}{*}{0.0005} \\
\hline & Negative) & $3(\mathrm{FN})$ & $68(\mathrm{TN})$ & 71 & \\
\hline & Total & 30 & 77 & 107 & \\
\hline \multirow{3}{*}{$>50$ years } & Positive & 17 (TP) & $3(\mathrm{FP})$ & 20 & \multirow{3}{*}{0.0005} \\
\hline & Negative & $3(\mathrm{FN})$ & $59(\mathrm{TN})$ & 62 & \\
\hline & Total & 20 & 62 & 82 & \\
\hline Sensitivity & $=27 / 30$ & $=90 \%$ & Sensitivity & $=17 / 20$ & $=85 \%$ \\
\hline Specificity & $=68 / 77$ & $=88.3 \%$ & Specificity & $=59 / 62$ & $=95.2 \%$ \\
\hline PPV & $=27 / 36$ & $=75 \%$ & PPV & $=17 / 20$ & $=85 \%$ \\
\hline NPV & $=68 / 71$ & $=95.8 \%$ & NPV & $=59 / 62$ & $=95.2 \%$ \\
\hline Accuracy & $=27+68 / 107$ & $=88.7 \%$ & Accuracy & $=17+59 / 82$ & $=92.6 \%$ \\
\hline
\end{tabular}

PPV: positive predictive value; NPV: negative predictive value; CT: computed tomography; SAH: subarachnoid haemorrhage.

Similarly, accuracy was observed above $80 \%$ for males and above $90 \%$ for females as shown in Table 3 . 
Table 3. Diagnostic accuracy of ct scan to diagnose subarachnoid hemorrhage in patients presenting with thunderclap headache by gender.

\begin{tabular}{llllll}
\hline \multirow{2}{*}{ Gender } & \multirow{2}{*}{ CT Scan } & Gold Standard LUMBER PUNCTURE & \multirow{2}{*}{ Total } & \multirow{2}{*}{ P-Value } \\
\cline { 3 - 4 } & Positive & Negative & 28 & 0.0005 \\
Male & Positive & $22(\mathrm{TP})$ & $6(\mathrm{FP})$ & 54 & \\
& Negative & $5(\mathrm{FN})$ & $49(\mathrm{TN})$ & 82 & 0.0005 \\
& Total & 27 & 55 & 79 & \\
Female & Positive & $22(\mathrm{TP})$ & $6(\mathrm{FP})$ & 107 & \\
& Negative & $1(\mathrm{FN})$ & $78(\mathrm{TN})$ & & \\
For Male & Total & 23 & 84 & $=22 / 23$ & $=95.7 \%$ \\
Sensitivity & & & For Female & $=78 / 84$ & $=92.9 \%$ \\
Specificity & $=22 / 27$ & $=81.5 \%$ & Sensitivity & $=22 / 84$ & $=78.6 \%$ \\
PPV & $=49 / 55$ & $=89.1 \%$ & Specificity & $=78 / 84$ & $=98.7 \%$ \\
NPV & $=22 / 28$ & $=78.6 \%$ & PPV & $=22+78 / 107$ & $=93.4 \%$ \\
Accuracy & $=49 / 54$ & $=90.7 \%$ & NPV & Accuracy & \\
\hline
\end{tabular}

PPV: positive predictive value; NPV: negative predictive value; CT: computed tomography; SAH: subarachnoid haemorrhage.

\section{Discussion}

Investigation of neurologically intact (i.e., no neurologic deficits) patients with headache is a potentially difficult clinical decision for physicians. Headache accounts for approximately $2 \%$ of all emergency department visits, and $\mathrm{SAH}$ is one of the most serious diagnosis, accounting for only $1 \%$ to $3 \%$ of these headaches [12-13]. TCH is head pain that begins suddenly and is severe at onset [14]. It refers to an excruciating headache of instantaneous onset [15]. It's an uncommon type of headache, but recognition and diagnosis are important because of the possibility of a serious underlying brain disorder. Most importantly, SAH should be excluded [9]. Patients suspected of having SAH are typically evaluated with an unenhanced CT scan followed by an LP if results of the CT scan are negative. CT is highly sensitive when performed soon after headache onset [5]. LP can be a painful procedure and can result in a headache that may be worse than the original headache [16]. The incidence of SAH has not diminished over time and is roughly 10 per 100,000 of the population and more common in Blacks and Hispanics than Whites [17-18]. Women, especially post-menopause, are more frequently affected than men [19-20]. Some studies suggest a rising incidence in elderly patients, and a decreasing incidence in men $[19,21]$. The average age in our study population was $47.97 \pm 9.96$ years. Out of 189 cases, 82 (43.39\%) were males and 107 (56.51\%) were females.

As noted in this study, all the patients had a CT brain without contrast for evaluation of $\mathrm{SAH}$ and subsequently underwent CSF analysis. Presence of SAH was confirmed by CSF analysis after LP. Many studies have attempted to quantify the value of the CT/LP approach in patients with suspected SAH; finding rates of confirmation by LP (after normal or equivocal CT results) were between $2.5 \%$ to $3.5 \%$ [22]. In this study, CT scan finding for SAH was positive in $29.6 \%(56 / 189)$ cases while LP analysis of the blood finding for SAH was positive in $26.46 \%(50 / 189)$ cases. In all but a single published case series, LP was required to diagnose SAH in a substantial number of cases in which CT was falsely negative [23-24]. LP adds useful clinical data, including the ability to differentiate among other diagnoses that present as severe headache, such as meningitis, idiopathic intracranial hypertension, venous sinus thrombosis, and spontaneous intracranial hypotension [11].

In a few studies done in the 1980s and 1990s, it was shown that $\mathrm{CT}$ alone had insufficient sensitivity for the detection of hemorrhage and would produce an unacceptable number of false-negative results [11, 24-25]. These older studies reported the sensitivity of CT scanning to be between $93 \%$ and $95 \%$ when done within 24 hours after symptom onset. The sensitivity declines to $85 \%$ after three days and to as low as $50 \%$ after a week [24-25]. LP has the advantage of picking up cases missed by CT, owing to its ability to detect small bleeds directly from CSF $[11,25]$. It remains the goldstandard diagnostic test [26]. Modern CT scanners are more sensitive detectors of SAH with reports of $100 \%$ sensitivity up to five days after symptom onset and an overall sensitivity of $99.7 \%$ (95\% CI: $98.1 \%-99.9 \%)$ and specificity of $100 \%$ (98.2\%-100\%) [27]. Despite the high sensitivity and specificity, suspected SAH with a negative CT scan still requires LP to confirm the absence of SAH [27]. Other studies report that $\mathrm{CT}$ sensitivity is slightly lower, between $95 \%$ and $100 \%$ on the first day, falling to about $58 \%$ at five days and less than 50\% after one week [28]. By day 10, the blood may have been totally resorbed. Some controversy exists about the utility of LP if CT scans are negative for SAH within six hours of ictus [23]. A more recent study by Backes D et al., completed in 2012, found that the sensitivity of head CT within six hours of ictus was $98.5 \%$ (95\% CI: 92.1\%-100\%), diagnosing all patients with aneurysmal and perimesencephalic SAH; sensitivity of CT performed after six hours was only $90.0 \%$ (95\% CI: 76.3-97.2) [29]. In this study sensitivity, specificity, PPV and NPV of CT was $88 \%$, $91.4 \%, 78.6 \%$, and $95.5 \%$ respectively while diagnostic accuracy of CT scan in the detection of SAH was $90.48 \%$ $(171 / 189)$.

\section{Conclusions}

This study concludes that CT is the preferred non-invasive approach for the majority of patients who present with suspected SAH. Its use is recommended in all patients of thunder clap headache as a part of the routine investigation. 
The study reaffirms that it is extremely sensitive if it is performed early and shows slight increased sensitivity in older patients however no sex prepondernace is seen. When all factors are considered, including its high sensitivity and wide availability, CT remains a time-honored and effective approach that should be valued and used by emergency physicians appropriately.

\section{References}

[1] Al-Shahi R, White PM, Davenport RJ, Lindsay KW: Subarachnoid haemorrhage. BMJ. 2006, 27:235-40.

[2] Linn FH: Primary thunderclap headache. HandbClin Neurol. 2010, 97:473-81. 10.1016/S0072-9752 (10)97042-5

[3] Coutinho JM, Stam J: A rare cause of thunderclap headache. BMJ. 2011, 341:6054. 10.1136/bmj.c6054

[4] Dodick DW: Thunderclap headache. J NeurolNeurosurg Psychiatry. 2002, 72:6-11.

[5] Perry JJ, Stiell IG, Sivilotti ML, et al.: Sensitivity of computed tomography performed within six hours of onset of headache for diagnosis of subarachnoid haemorrhage: prospective cohort study. BMJ. 2011, 18:4277. 10.1136/bmj.d4277

[6] O'Neill J, McLaggan S, Gibson R: Acute headache and subarachnoid haemorrhage: a retrospective review of CT and lumbar puncture findings. Scot Med J. 2005, 50:151-3. 10.1177/003693300505000405

[7] Mehrotra P, Sookhoo S, Kolla S, Halbert H, Lavell K, England S: Investigation of subarachnoid haemorrhage: does the buck stop with CT? J Med Life. 2010, 15:338.

[8] Ju YE, Schwedt TJ: Abrupt-onset severe headaches. Semin Neurol. 2010, 30:192-200.

[9] Hankey GJ, Nelson MR: Subarachnoid haemorrhage. BMJ. 2009, 21:2874. 10.1136/bmj.b2874

[10] Farzad A, Radin B, Oh JS, et al.: Emergency diagnosis of subarachnoid hemorrhage: an evidence-based debate. J Emerg Med. 2013, 1:1045-53. 10.1016/j.jemermed.2012.10.001

[11] Mann D: The role of lumbar puncture in the diagnosis of subarachnoid hemorrhage when computed tomography is unavailable. Can J Emerg Med. 2002, 4:102-5. $10.1017 / \mathrm{S} 1481803500006205$

[12] Edlow JA, Panagos PD, Godwin SA, Thomas TL, Decker WW: Clinical policy: critical issues in the evaluation and management of adult patients presenting to the emergency department with acute headache. J EmergNurs. 2009, 1:43-71. 10.1016/j.jen.2008.12.009

[13] Perry JJ, Stiell I, Wells G, Spacek A: Diagnostic test utilization in the emergency department for alert headache patients with possible subarachnoid hemorrhage. Can J Emerg Med. 2002, 4:333-7. 10.1017/S1481803500007739

[14] Schwedt TJ, Matharu MS, Dodick DW: Thunderclap headache. Lancet Neurol. 2006, 5:621-31. 10.1016/S14744422 (06)70497-5
[15] Ducros A, Bousser MG: Thunderclap headache. BMJ. 2013, 9:8557. 10.1136/bmj.e8557

[16] Evans RW, Armon C, Frohman EM, Goodin DS: Assessment: prevention of post-lumbar puncture headaches. Neurology. 2000, 55:909-14. 10.1212/WNL.55.7.909

[17] Labovitz DL, Halim AX, Brent B, Boden-Albala B, Hauser WA, Sacco RL: Subarachnoid hemorrhage incidence among whites, blacks and Caribbean Hispanics: the northern Manhattan study. Neuroepidemiology. 2006, 26:147-50. $10.1159 / 000091655$

[18] Rothwell PM, Coull AJ, Giles MF, et al.: Change in stroke incidence, mortality, case-fatality, severity, and risk factors in Oxfordshire, UK from 1981 to 2004 (Oxford Vascular Study). The Lancet. 2004, 12:1925-33. 10.1016/S0140-6736 (04)16405-2

[19] Stegmayr B, Eriksson M, Asplund K: Declining mortality from subarachnoid hemorrhage: changes in incidence and case fatality from 1985 through 2000. Stroke. 2004, 1:2059-63. 10.1161/01.STR.0000138451.07853.b6

[20] Nilsson OG, Lindgren A, Ståhl N, Brandt L, Säveland H: Incidence of intracerebral and subarachnoid haemorrhage in southern Sweden. J NeurolNeurosurg Psychiatry. 2000, 1:6017. 10.1136/jnnp.69.5.601

[21] Inagawa $\mathrm{T}$ : Trends in incidence and case fatality rates of aneurysmal subarachnoid hemorrhage in Izumo City, Japan, between 1980-1989 and 1990-1998. Stroke. 2001, 32:1499507.

[22] Van der Wee N, Rinkel GJ, Hasan D, Van Gijn J: Detection of subarachnoid haemorrhage on early CT: is lumbar puncture still needed after a negative scan?. J NeurolNeurosurg Psychiatry. 1995, 58:357-9. 10.1136/jnnp.58.3.357

[23] Boesiger BM, Shiber JR: Subarachnoid hemorrhage diagnosis by computed tomography and lumbar puncture: are fifth generation CT scanners better at identifying subarachnoid hemorrhage?. J Emerg Med. 2005, 1:23-7. 10.1016/j.jemermed.2005.02.002

[24] Van Gijn J, Van Dongen KJ: The time course of aneurysmal haemorrhage on computed tomograms. Neuroradiology. 1982, 23:153-6.

[25] Mushtaq SA, Bodla MA: Diagnostic accuracy of computed tomography for subarachnoid haemorrhage in patients presenting with thunderclap headache (Lumbar Puncture as Gold Standard). Pak J Med Health Sci. 2014, 8:

[26] Cortnum S, Sørensen P, Jørgensen J: Determining the sensitivity of computed tomography scanning in early detection of subarachnoid hemorrhage. Neurosurgery. 2010, 66:900-3. 10.1227/01. NEU.0000367722.66098.21

[27] Brown SC, Brew S, Madigan J: Investigating suspected subarachnoid haemorrhage in adults. BMJ. 2011, 6:2644. 10.1136/bmj.d264

[28] Backes D, Rinkel GJ, Kemperman H, Linn FH, Vergouwen MD: Time-dependent test characteristics of head computed tomography in patients suspected of nontraumatic subarachnoid hemorrhage. Stroke. 2012, 43:2115-9. 10.1161/STROKEAHA.112.658880 